\title{
Quistes ováricos en perras: condición multifactorial y de difícil tratamiento
}

Laura Castro-Ramírez ${ }^{1} \bowtie$, Isabel Hagnauer-Barrantes², Alejandro Alfaro-Alarcón ${ }^{3}$, Marcela Suárez-Esquivel ${ }^{1}$

1 Programa Integrado de Endocrinología y Reproducción Animal, Escuela de Medicina Veterinaria (EMV), Universidad Nacional (UNA), Costa Rica. lcastro@una.cr, marcela.suarez.esquivel@una.cr

2 Hospital de Especies Menores y Silvestres, Escuela de Medicina Veterinaria, Universidad Nacional, Costa Rica. isabel.hagnauer.barrantes@una.cr

3 Laboratorio de Patología, Programa Hospital, Escuela de Medicina Veterinaria, Universidad Nacional, Costa Rica. alejandro.alfaro.alarcon@una.cr

Los quistes ováricos en perras son una condición poco frecuente, multifactorial y de difícil tratamiento, condición que adquiere relevancia ante el aumento de la popularidad en la reproducción de determinadas razas con fines comerciales. Una perra American Strafford de 8 años, con historia de haber estado extraviada durante un mes aproximadamente, ingresó por un cuadro de desnutrición severa, al examen físico se observó secreción vulvar purulenta, y por esto se realizó una evaluación ultrasonográfica abdominal que evidenció contenido intrauterino. La paciente no presentaba conducta sugestiva de actividad estrogénica elevada. Las pruebas de laboratorio mostraron anemia no regenerativa, trombocitopenia e hipoalbuminemia. Aun así, se sometió a ovario-histerectomía ante el riesgo de la infección. La inspección del tracto reproductivo extraído, mostró un ovario izquierdo de grandes dimensiones $(2.5 \times 4 \mathrm{~cm})$, con apariencia irregular multinodular y presencia de varios quistes, con tamaños entre 0.2 a $0.5 \mathrm{~cm}$ de diámetro con contenido acuoso translúcido. El análisis histopatológico del ovario reveló dos cuerpos lúteos, uno de ellos con una dilatación quística, además de folículos primordiales, primarios en crecimiento y terciarios. En el nivel de la médula ovárica, originándose de la rete ovarii, se observó una proliferación neoplásica con múltiples estructuras quísticas, mientras que, en otros puntos, la proliferación neoplásica presentaba un crecimiento arboriforme (cistoadenoma papilar ovárico). Adicionalmente, el útero presentaba hiperplasia endometrial quística. Se realizó medición de hormonas reproductivas en el fluido quístico y en suero. En el valor sérico se detectó una concentración de progesterona de $4.19 \mathrm{ng} / \mathrm{ml}$, testosterona de $13.01 \mathrm{ng} / \mathrm{dl}$ y estradiol $<25 \mathrm{pg} / \mathrm{ml}$. En el fluido quístico se obtuvo una concentración de $29.24 \mathrm{ng} / \mathrm{ml}$ de progesterona, $50 \mathrm{pg} / \mathrm{ml}$ de estradiol y $51.45 \mathrm{ng} / \mathrm{dl}$ de testosterona. Este caso evidencia la complejidad de la patogénesis de los síndromes poliquísticos y del abordaje terapéutico en hembras destinadas a la reproducción, ya que su origen y estructura es multifactorial.

凶 Autor para correspondencia Laura Castro-Ramírez: lcastro@una.cr 